\title{
Los problemas
}

de la universidad liberal

según Alasdair Maclntyre ${ }^{1}$

\author{
(The problems \\ of the liberal university \\ according to Alasdair Maclntyre)
}

\section{JOSÉ MANUEL GIMÉNEZ AMAYA}

Grupo Ciencia, Razón y Fe (CRYF)

Universidad de Navarra, Pamplona, España

jmgimenezamaya@unav.es

ORCID: 0000-0002-6977-3963

\section{SERGIO SÁNCHEZ-MIGALLÓN}

Grupo Ciencia, Razón y Fe (CRYF)

Universidad de Navarra, Pamplona, España

smigallon@unav.es

ORCID: 0000-0002-7295-2735

Resumen. La universidad que Alasdair MacIntyre llama liberal es aquella que rechaza la tradición como algo premoderno y como un impedimento para el progreso, también pedagógico. Este modelo de universidad se ha generalizado en nuestros días, caracterizándose por dar prioridad a la formación técnica y pragmática, en perjuicio de una visión global y sapiencial. Esta última es la clave para unificar el saber, para trasmitirlo como tal, y para, por tanto, formar integralmente a las personas. Sin embargo, la profe-

1 Este trabajo está dedicado al Profesor Alasdair MacIntyre en su 90 cumpleaños (2019).

Scientia etFides 8(1)/2020, 99-121 
sionalización de la filosofía y los criterios del liberalismo moderno parecen dominar el espíritu de las universidades actuales, sin que sean capaces de advertir -engañándose en una retórica ingenua - su incapacidad de formar de manera sapiencial y moral a sus profesores y estudiantes.

Palabras clave: universidad; liberalismo; tradición; formación; filosofía.

Abstract. The university that Alasdair Maclntyre defines as liberal is that which rejects the tradition as something pre-modern and with an impediment for the progress, including the pedagogic one. This university model has been extended in all instances, giving priority to the technical education in a pragmatic way. Thus, the global and sapiential vision of the university has been relegated. This last approach is the key for unifying the knowledge and to pass it onto other people and, therefore, to educate and mold intellectually and morally. However, the professionalization of the philosophy and the criteria of the modern liberalism seem to dominate and inspire most universities in the present day. This situation has plainly shown an inability to become aware of their incompetence to properly educate professors and students alike, in a sapiential and moral manner, threfore deceiving themselves with a naive rhetoric.

Keywords: university; liberalism; tradition; formation; philosophy.

El presente texto sigue las ideas generales que ya expusimos en nuestro libro Diagnóstico de la Universidad en Alasdair MacIntyre: génesis y desarrollo de un proyecto antropológico (Giménez Amaya y Sánchez-Migallón 2011). Sin embargo, estos años pasados desde la publicación de nuestro texto nos han permitido reflexionar más en profundidad sobre lo escrito, especialmente sobre las palabras del propio MacIntyre, también en el contexto de su obra publicada posteriormente, Ética en los conflictos de la modernidad: sobre el deseo, el razonamiento práctico y la narrativa (MacIntyre 2016; Bello y Giménez Amaya 2018). Todo apunta a que lo que él denominó universidad liberal se ha implantado de forma generalizada y casi universal en la enseñanza superior y esto, dentro de una sociedad moderna que la quiere, acoge y necesita, aunque no sabe dar respuesta en profundidad al por qué ni al para qué de su presencia y desarrollo.

De una forma general, y en el marco de su obra After Virtue: A Study of Moral Theory (MacIntyre 1984; 1987a) -que al mismo tiempo concibe como un proyecto a desarrollar, proyecto After Virtue-, MacIntyre resalta la universidad liberal como una comunidad universitaria que desacredita todo ethos y, en definitiva, toda tradición configuradora diferente de lo 
establecido por el liberalismo (Ruiz Arriola 2000,78). Como crítica a esto último, el propio MacIntyre se ha planteado de manera problemática, y con muchas reservas, adjudicar al liberalismo, en sí mismo, el estatuto de tradición (Giménez Amaya y Sánchez-Migallón 2011, 146-161).

El liberalismo se define como ausencia de un acuerdo sobre cuál es el bien humano para el hombre (Ruiz Arriola 2000, 82). Y esto último es, justamente, el punto nuclear para entender cualquier tradición. La falta de acuerdo en el liberalismo origina una proliferación de bienes heterogéneos en distintas áreas de la vida humana y la aparición de un «yo» emotivo, que resulta central para entender el panorama moral contemporáneo. Pretender caracterizar el liberalismo como tradición puede ser contradictorio, porque ello implicaría que existe una noción de bien que actúa a modo de criterio para organizar, ordenar y elegir entre los distintos bienes particulares; criterio que se propone para ser aplicado y transmitido. Pero esto vemos que no sucede en el liberalismo (Rodríguez Duplá 2000, 31-43; Giménez Amaya y Sánchez-Migallón, 2011, 152-156).

Siguiendo ahora con el problema del pensamiento liberal, en cuanto a nuestro tema se refiere, es objetivo reconocer que, de algún modo, en la sociedad actual los valores del liberalismo se pueden considerar, de hecho, análogos a los del mercado. Esto impide de forma perniciosa que se suscite un pensamiento crítico, un pensamiento por sí mismo. Para MacIntyre, pensar por sí mismo es sinónimo de saber más allá de las normas aprendidas de la tradición en la que uno se ha formado, o saber extrapolar las reglas conocidas en nuestro aprendizaje a nuevas situaciones que no se habían previsto, reguladas ahora por las propias contingencias de la vida. No tiene nada que ver, por tanto, con el solipsismo o con una racionalidad instrumental, que es como la tradición liberal entiende esa independencia de juicio (MacIntyre 1987a, 172-187).

En la actualidad, el problema reside en que la propia apertura universalista de la academia desacredita en su esencia las tradiciones que contienen valores diferentes. La universidad de corte liberal se encuentra con que no puede, e incluso impide, acceder a lo más alto de la capacidad intelectual, a ese lugar al que sólo se puede llegar pensando por sí mismo. 
Es claro que, en este contexto, los presupuestos, actividades y objetivos de la universidad liberal no pueden adecuarse de forma diáfana a lo que en un principio concibió la Ilustración que debía ser. Pues no se ha llegado a un acuerdo sobre cuáles deberían ser los principios universales que deberían informarla. Se repiten aquí muchos de los aspectos que MacIntyre discute en su obra y proyecto After Virtue con respecto a los debates morales contemporáneos. Todo ello ya se ha presentado y discutido en detalle en un trabajo nuestro anterior (Giménez Amaya y Sánchez-Migallón 2011, 134-163).

Para MacIntyre, el fracaso del proyecto ilustrado hace necesario también replantearse el fin de la universidad, y que esta institución capital de la formación en la sociedad tenga carta de naturaleza en las grandes discusiones sobre el desengaño de la filosofía de la modernidad y de la posmodernidad (MacIntyre 2012, 207-281). Para Long, si este debate no se lleva a cabo, la propia universidad y la sociedad entera corren el riesgo de sucumbir a la lógica de un utilitarismo mercantilista que embarga las relaciones sociales y el pensamiento actual. En sus propias palabras, que traducimos del inglés, nos dice este autor: «¿Para qué es el conocimiento? Las instituciones que persiguen sus objetivos sin jamás preguntárselo venden, por lo general, su saber al mejor postor [...] en tanto la sociedad en torno a ellas se vuelve más precaria por falta de una guía responsable» (Long 1992, 181). MacIntyre va a argumentar que lo que Long aún ve como una amenaza, ha sucedido ya, desgraciadamente, tanto en la universidad como en la sociedad en que vivimos.

Pero acudamos otra vez a preguntarnos por el diagnóstico que hace MacIntyre de la universidad liberal. Nuestro autor sigue la idea de que, para comprender las instituciones de una sociedad, es preciso preguntarnos por la sociedad misma (MacIntyre 1987b, 25). Para el filósofo británico, el cambio de la universidad es consecuencia directa del desarrollo del liberalismo, sobre todo en el ámbito del proyecto ilustrado de finales del siglo XVIII (MacIntyre 1992, 268).

Como ha sugerido con agudeza nuestro autor en el desarrollo de su proyecto After Virtue, el orden moderno ha fragmentado el concepto de verdad. La educación se ve enfrentada con una alternativa en la que es posible educar profesionalmente con cierta eficacia, pero no en aspectos 
fundamentales de la existencia, como es el caso de la sabiduría práctica que nos permita elegir críticamente el mejor tipo de vida que nos permita ser buenos y felices (Ruiz Arriola 2000, 118).

Es este un punto medular de la evaluación de nuestro autor sobre la enseñanza. Para él, sin la integridad de la formación en la verdad, la sabiduría se ve excluida de los objetivos pedagógicos institucionales, y la educación se ve ante una disyuntiva en la que se debe elegir entre la formación cultural -que también podemos entender desde una perspectiva más sapiencial- o el entrenamiento (práctico) profesional. La modernidad ha provocado una escisión otorgando a cada uno de esos caminos una noción de verdad y de vida buena incompatibles entre sí. Esta esquizofrenia intelectual tiende a romperse con facilidad. La vida buena, la formación en virtudes, queda aherrojada o subyugada a una quimera imposible de lograr de forma colectiva. Igualmente, se implanta un individualismo moral que centra su actividad en un aprendizaje fragmentado que hace imposible dar coherencia unitaria a nuestro saber. Así dice MacIntyre en traducción del inglés realizada por Ruiz Arriola $(2000,118)$ :

El que estos dos objetivos generales sean mutuamente incompatibles, que el éxito de uno llevará al fracaso del otro, no es una tesis conceptual que siempre ha sido cierta. [...] de hecho, son las culturas y sociedades específicamente modernas, postilustradas, las que ahora excluyen las condiciones que hacían posible esta coexistencia (MacIntyre 1987b, 17).

Para MacIntyre, por tanto, el desarrollo de una universidad que afronte una visión sapiencial como base del aprendizaje se revela como algo crucial (Giménez Amaya 2012, 193-202). Pero su propuesta universitaria es radicalmente diferente a la de los ilustrados que viven en una especie de camuflaje moderno-posmoderno, muchas veces sin solución de continuidad. Esta propuesta la ha plasmado nuestro autor a lo largo de los años en los que desarrolla y aplica su proyecto After Virtue (Giménez Amaya y Sánchez-Migallón 2011, 15). Y se ha desplegado de forma bastante completa en los últimos años, donde se manifiesta su búsqueda de la formación sapiencial como eje de la vida universitaria. 
Pero analicemos esto con detenimiento siguiendo varios pasos. Lo primero que detecta MacIntyre es que la universidad liberal rechaza toda la teleología aristotélica y relega el estudio de la filosofía moral a los especialistas. Esto hace que sea difícil establecer unos parámetros alternativos. Pero, a pesar de ello, MacIntyre los expone nítidamente. Para él, la simple reforma curricular -es decir el cambio de los patrones en la formación y en la enseñanza en general- es claramente insuficiente. Se requiere favorecer la presencia de comunidades educativas que permitan una reorganización de la sociedad, que se encuentra sumamente fragmentada. Como condición, MacIntyre insistirá en la participación, es decir, en un clima donde se puedan compartir las creencias y actitudes derivadas de las lecturas de los mismos textos interpretados a la luz de criterios de racionalidad homogéneos (MacIntyre 1987b, 19-25; Ruiz Arriola 2000, 119).

En opinión de MacIntyre, hace falta una universidad que comparta una tradición según unas creencias sustanciales, y no sólo un acuerdo metodológico o procedimental, que es como se articula mayoritariamente la formación universitaria en nuestros días. Además, su crítica a la universidad liberal se sustancia en su función social. Así, él propone que se pueda realizar un acuerdo fundamental para impulsar un debate sobre las cuestiones de peso para la vida individual, y para el logro de soluciones congruentes con el verdadero bien humano de cada una de las personas y de la comunidad (MacIntyre 1987b, 15-36; 1992, 267-289).

\section{Una universidad que admite la fragmentación del saber}

Una primera aclaración. Cuando se habla aquí de fragmentación queremos indicar la ausencia de relación entre los saberes. Sobre todo en lo referente a la intención de adquirir un conocimiento que está integrado y conectado con una realidad unitaria y llena de sentido. Lógicamente, el acceso a los saberes particulares, y a su conocimiento en profundidad, exige una cierta fragmentación, que más bien podría definirse también como una concentración o distinción, que es lo que permite poder estructurar el conocimiento en profundidad de las diferentes ciencias. 
Uno de los aspectos en los que más insiste MacIntyre con respecto al enfoque de la enseñanza universitaria es la profunda pérdida de una concepción del mundo (Weltanschauung) que pueda justificarse racionalmente. De ahí la imposibilidad que hay para encontrar unos principios universales y, al mismo tiempo, el rechazo constante del concepto de tradición como portador de racionalidad (Lutz 2009, 33-63).

Merece la pena, por tanto, preguntar si la ilustración no habrá contribuido de un segundo modo a nuestra condición presente; no sólo por lo que sus logros al propagar sus doctrinas distintivas hayan efectuado, sino también por lo que ha conseguido ocultar de nuestra vida (MacIntyre 1994, 23).

En la cita anterior hemos resaltado en cursiva la palabra «ocultar», porque pensamos que la idea del ocultamiento en nuestro autor es muy acertada para describir las acciones morales en la sociedad liberal e ilustrada y MacIntyre la va a utilizar con frecuencia para resaltar las incoherencias que presenta esta sociedad y la universidad que se ampara en ella.

Aquello en que la ilustración nos ha cegado en gran parte, y que ahora tenemos que recuperar es, a mi juicio, una concepción de la investigación racional tal como se incorpora en una tradición, una concepción según la cual los criterios mismos de la justificación racional surgen y forman parte de una historia, en la que estos -los criterios- están justificados por el modo en que trascienden las limitaciones y proporcionan los remedios para los defectos de sus predecesores en la historia de la propia tradición (MacIntyre 1994, 24).

Con estos agudos comentarios MacIntyre apunta a la entraña del pensamiento universitario ilustrado (MacIntyre 1994, 7; Ruiz Arriola 2000, 121). No se trata tanto de que se asimile en bruto una gran cantidad de conocimientos, sino más bien de la total ausencia de un encuadre global -de una tradición, de un marco de referencia- donde insertarlos. El resultado no se ha hecho esperar: nos encontramos con una fragmentación del saber que se ha atomizado hasta la saciedad, donde reina un mosaico muy amplio de disciplinas autónomas y especializadas. Lo más grave de este problema, apunta MacIntyre con lucidez, es que estamos ante una crisis epistemológica 
en el corazón mismo de la universidad liberal, donde lo más serio y hasta dramático es que:

la universidad post-enciclopédica [...] se ha visto confrontada con cuestiones para cuya respuesta carece de recursos (MacIntyre 1992, 273).

Esta crisis, según nos explica el autor anglosajón, que lleva a una incapacidad de comprender la realidad, no depende de que tengamos que manejar una ingente cantidad de aspectos relevantes. Él mismo nos dice que:

A veces se otorga una importancia exagerada a la mera cantidad en el aumento del saber y se sugiere que la razón por la que ya no es posible un conocimiento general de las disciplinas es que simplemente hay demasiado saber. Pero la cantidad en sí no es muy importante (MacIntyre 1987b, 28; Ruiz Arriola 2000, 121-122).

El verdadero avance hay que buscarlo, según nuestro autor, en los caracteres cualitativos del saber (Llano 1988, 44). Dejemos que siga hablando MacIntyre con firmeza y luminosa visión:

Lo que es más importante es el tipo de profesionalización que hace del contenido especializado de cada disciplina materia de su investigación, pero excluye de cualquier disciplina los temas de relevancia de otras disciplinas (MacIntyre 1987b, 28; Ruiz Arriola 2000, 122).

Y para MacIntyre, el caso paradigmático ha sido la filosofía moral, completamente relegada en la educación superior liberal. El problema era que ésta, precisamente, proporcionaba el acuerdo más fundamental a toda la empresa educativa.

En realidad, este desacuerdo se amplía a toda la filosofía, de tal manera que la crisis hace imposible abrigar el proceso de racionalidad dentro de una unidad en la Weltanschauung que permita alcanzar un avance en la complejidad estructural del mundo de hoy. Para nuestro autor, sólo la filosofía puede proporcionar unas líneas maestras de racionalidad que faciliten integrar y descifrar los resultados de las distintas disciplinas 
que integran la universidad liberal. MacIntryre defiende que, si no hay un fundamento filosófico unitario, los problemas y las soluciones genéricas que la investigación descubre se contradicen y se anulan entre sí (MacIntyre 1983, 23). Muy bien se podría decir que, en este caso, el conocimiento más que esclarecer, ofusca; se pierde lo más propio de la universidad, que es el conocimiento unitario, universal.

Sea como fuere, la universidad para MacIntyre tiene una importancia decisiva en la sociedad actual. Dejada ésta al albur de la corriente liberal, con la consiguiente fragmentación en el pensamiento, se hace imposible la necesaria y urgente reconstrucción moral descrita en el proyecto After Virtue (Giménez Amaya y Sánchez Migallón 2011, 229-232). De ahí que el filósofo británico insista en que la misión de la universidad -función que ninguna otra institución en la sociedad puede desempeñar por ella- es la elaboración de

concepciones y criterios de justificación racional [...] [que guían] las detalladas prácticas de investigación, [...], de manera que sólo de la universidad puede aprender la sociedad en general cómo conducir sus propios debates, prácticos o teóricos, de un modo que pueda justificar racionalmente (MacIntyre 1992, 274).

La tesis de MacIntyre es, por tanto, que en el estado actual la universidad liberal no es una institución educativa en un sentido pleno y moral. Esa universidad liberal nace de un desacuerdo ilimitado de sus autores y se ha convertido en meras colecciones de datos y de hechos, ordenados muy sistemáticamente, para el único beneficio de la búsqueda de referencias (MacIntyre 1992, 268). En esto la universidad liberal tiende a ser lo que decía con perspicacia Becker sobre este tema: «Si se entendiera la ciencia únicamente como el proceso de acumular datos sin relación, la universidad perdería todo su significado educativo» (Becker 1994, 186).

\section{La desaparición de la Universitas}

Universitas es entendida, especialmente a partir del siglo XIII, como la comunidad de profesores y alumnos (universitas personarum) y, a su vez también, 
como el conjunto de los contendidos estudiados que configuran la enseñanza de la unidad del saber (universitas studiorum) (Martínez-Echevarría 2010, 63-66). Estas dos formas de entender este término son pertinentes en la manera en que Alasdair MacIntyre concibe la universidad.

En este contexto y como venimos diciendo, el resultado más notorio, para la universidad del cambio que se ha introducido con el fenómeno cultural de la Ilustración, lo va a situar MacIntyre en la fragmentación y especialización de las distintas disciplinas de estudio, con su correspondiente organización de las diferentes ramas del saber. La unidad del saber se resume, casi únicamente, en compartir un lugar físico más o menos cercano. Según MacIntyre:

[...] nada es más llamativo en la universidad contemporánea que la extensión de las divisiones y de los conflictos - que continúan, a lo que parece, de manera ineludible- en el seno de toda la investigación humanística [...] (MacIntyre 1992, 29).

La universidad fue inicialmente una comunidad que incluía a maestros y a estudiantes que se unían en el compromiso de avanzar en el saber (Bowen 1986, 161-165; Saranyana 2007, 230-232; Winchester 1986, 269-290). Sin embargo, se ha visto transformada en el curso de los siglos en lo que el reconocido profesor norteamericano Clark Kerr ha denominado «una colección de comunidades y actividades unidas bajo un mismo nombre» (Kerr 1963, 1) $)^{2}$.

La pérdida de estos principios fundacionales en la comunidad universitaria es, para MacIntyre, un proceso largo y complicado que se desarrolla a lo largo de los siglos, en el que influye de manera importante el cambio operado en la filosofía. En el pensamiento de nuestro autor no existe una narrativa explicativa simplista de causa-efecto, sino que, más bien, él describe una visión que se puede aplicar a la universidad y a la sociedad en

2 Clark Kerr (1911-2003) es un conocido profesor norteamericano de Economía, que también fue el duodécimo presidente de la Universidad de California. El texto al que nos referimos en nuestro estudio recoge la reconocida Edwin L. Godkin Lecture de este autor, que impartió en la Universidad de Harvard en el año 1963. 
general: se trata de una sucesión de fenómenos que son, al mismo tiempo, causa y efecto (Ruiz Arriola 2000, 124).

Sin embargo, es evidente que el proceso de la Ilustración marca, para MacIntyre, un punto de inflexión en el desarrollo de la filosofía moral, que influye en la formación universitaria. Para nuestro autor, todo conocimiento adquiere su justificación en cuanto contribuye a hacer posible una vida buena. Según él, el artificio liberal de partir de unos principios morales mínimos no sólo ha empobrecido a la misma filosofía moral, sino que el propio quehacer científico de la universidad se ha visto profundamente restringido de los criterios racionales de verdadero progreso, e incluso de un concepto unívoco de qué es aquello que lo constituye (MacIntyre 1992, 34).

Es interesante ver cómo MacIntyre asocia la pérdida de la noción de investigación universal, de formación en un saber universal, a la pérdida de los criterios racionales para determinar la vida buena para el hombre. Justamente al haberse pérdido el concepto de vida buena, el horizonte racional se hace verdaderamente imposible para determinar el valor real de un hallazgo, de cualquier innovación, descubrimiento o invento. Se ha destruido el intento de crear una educación en la comunidad al margen de cualquier tradición, que ahora se hace piedra angular de la propia educación liberal.

Los resultados son demasiado manifiestos como para obviarlos. En palabras de Bell, la clave de la educación contemporánea reside en la «exigencia fundamental [de la educación moderna que] es ser “objetivo” en la formulación de las respuestas: reducir las "parcialidades” que derivan de una educación parroquial, eliminar los prejuicios y la superstición, renunciar a la tradición y al egoísmo, etc. Para ser objetivos, es menester "purificar" las ideas [...], y para ser éticamente racionales, es necesario "universalizar” la propia conducta en la forma de un imperativo categórico» (Bell 1992, 35). Parece claro que uno de los pilares básicos de la universidad liberal es su animadversión a los prejuicios, a lo contextual y a la tradición, y esos prejuicios se han trasladado a la arquitectura formativa y configurativa de la sociedad moderna (Ruiz Arriola 2000, 125).

Vemos, por tanto, que en la pérdida del sentido de comunidad y tradición está la insuficiencia, en una gran parte, de los acuerdos que refundan la 
universidad liberal. Al fracaso consumado para alcanzar un acuerdo racional sobre los principios que fundan la moralidad, ha seguido necesariamente la proliferación de facciones y la atomización de la propia comunidad universitaria (Ruiz Arriola 2000, 126). Esto se ha llevado a cabo de modo muy paradigmático en la escisión de la comunidad universitaria en dos ámbitos culturales que se alejan cada vez más entre sí: el humanista y el científico experimental. A esto hay que añadir también, según Kerr, el desarrollo de los estudios sobre las ciencias económicas y sociales (Kerr 1963, 119). Sobre esta escisión, los desacuerdos han seguido creciendo, y ha habido que dividir todavía más los saberes en sub-disciplinas y sub-especialidades, que intentan abarcar ámbitos del conocimiento de la realidad aún más concentrados y limitados.

Para MacIntyre, sin una comunidad que educa y forma en una tradición, se diluye la unidad del conocimiento y la posibilidad de una comprensión global del mundo que nos rodea. Esa es la verdadera función de la universidad. Como nos recuerda el autor británico, el origen de esta última surge de la necesidad de dar una formación general a las personas que se encuentran con la tarea comunitaria de sustentar la vida intelectual y moral en esa sociedad (MacIntyre 1987b, 15-36; Giménez Amaya y Sánchez-Migallón 2011, 246-254). De esta manera, por ello, según Bowen, la universidad debe «[su] aparición [...] a las imperiosas necesidades de dar una formación adecuada a juristas, maestros y clérigos» (Bowen 1986, 156). Y comenta Ruiz Arriola que «[el] núcleo de los estudios superiores al inicio de la universidad eran las Artes Liberales que, tanto en París como en Bolonia pese a las divergencias en cuanto al fin del conocimiento, se estudiaban bajo los mismos presupuestos racionales de interpretación que servían de base al debate de las quaestiones disputatae y las más generales quaestiones quodlibetales, los métodos pedagógicos por excelencia de esa época y que no difieren en nada de la dialéctica propuesta por MacIntyre cuyo modelo paradigmático es precisamente la Universidad de París en el siglo XIII» (Ruiz Arriola 2000, 126-127).

MacIntyre también ha estudiado la universidad medieval (MacIntyre 2012, 121-158) y se une a un grupo de pensadores que piensan que, al cerrarse la universidad liberal a las disciplinas que dan unidad al universo 
del saber, ésta queda únicamente como una institución que prepara especialistas para adentrarse en un mundo mercantilista (Bowen 1986, 166-190; MacIntyre 1992, 166-190).

En este contexto unitario, nuestro autor da especial importancia a la filosofía y a la teología. Es pertinente aquí recordar las conocidas palabras del pensador alemán Josef Pieper, con las que ciertamente no puede menos que coincidir MacIntyre, señalando que: «cuando se habla del lugar y de la justificación de la filosofía se trata, nada más ni nada menos, que del lugar y de la justificación de la universidad, de la formación académica, mejor dicho de la formación en general en sentido auténtico; es decir, en el sentido en que se distingue primariamente de cualquier mera instrucción profesional y la supera» (Pieper 1983, 36).

En resumen, la pérdida de esta visión universalista, de la tradición y de la concepción de la vida buena para el hombre, ha hecho para MacIntyre que la universidad pierda su razón de ser, o que, mejor dicho, adquiera otra $\mathrm{u}$ otras funciones, aun cuando en apariencia siga justificándose con los mismos objetivos originales de racionalidad.

El grado en que es difícil prever la reestructuración de la universidad [para que pueda cumplir su misión como un foro de debate entre tradiciones de justificación racional opuestas y diferentes de la vigente] [...] es también el grado en que las estructuras de la sociedad presente se han eximido y se han protegido de ser puestas en cuestión por semejante investigación intelectual y moral sistemática (MacIntyre 1992, 289).

\section{La profesionalización de la filosofía}

¿Y qué ha pasado en este nuevo contexto con la filosofía en la universidad? La filosofía ha sufrido un proceso de especialización que ha visto su mayor auge y expresión en el gran avance de la corriente analítica. Lo más llamativo para nuestro autor es que el ascenso de esta forma de enfocar los estudios filosóficos se ha visto acompañada de la decadencia de la obra de Tomás de Aquino, fruto de una mala interpretación. Porque, para el filósofo británico, 
la obra de este autor medieval es la mejor síntesis en la investigación moral de toda la historia del pensamiento (MacIntyre 1987a, 207-225).

Nuestro autor ha criticado mucho esta profesionalización de la filosofía. Un botón de muestra es lo que escribe Turner sobre el tema: «La caracterización que MacIntyre hace de la filosofía como una labor artesanal parece estar en tensión con su dura crítica de la filosofía analítica» (Turner 1991, 272; Ruiz Arriola 2000, 129). A lo que comenta agudamente Ruiz Arriola: «Sin duda es sencillo explicar la animosidad de MacIntyre hacia la filosofía analítica, en primer lugar porque constituye el reverso de la moneda de la decadencia de la filosofía moral y se deriva de la inconmensurabilidad de las posiciones éticas surgidas a raíz del rechazo moderno de la teleología» (Ruiz Arriola 2000, 129-130).

Quizá lo crucial en la crítica de MacIntyre a la filosofía analítica se concentre en que esta última olvida, de su horizonte de objetivos, la pregunta ética de la vida buena para el hombre. En un contexto más pragmático, se podría decir que, tal como se viene practicando este tipo de hacer filosofía, los resultados de su investigación no se plasman en la vida de los individuos.

En el fondo, para MacIntyre, esta preeminencia de la corriente analítica en nuestros días es una forma más de supervivencia de la filosofía, en el mundo moderno e ilustrado que ha establecido las bases de qué debe y qué no debe ser considerado como científico. En otras palabras, la propia filosofía no quiere verse relegada a la ignorancia y al olvido, como lo ha sido la teología en el ambiente ilustrado de la modernidad. Aquella, por tanto, ha seguido una deriva que la ha llevado a la asunción de que no debe llegar a ningún acuerdo en lo fundamental.

Lo que el progreso de la filosofía analítica ha logrado establecer es que no hay bases para creer en principios universales necesarios, sino sólo relativos a un conjunto de presuposiciones (MacIntyre 1989, 414; Ruiz Arriola 2000, 130).

Para nuestro autor, la filosofía analítica requiere únicamente un mínimo acuerdo procedimental para

dilucidar una multitud de relaciones lógicas y conceptuales (MacIntyre 1992, 36). 
La abierta oposición de MacIntyre hacia la forma de hacer filosofía de los analíticos, y, en general, a la forma en que se procura reducir las humanidades en muchas universidades anglosajonas a procedimientos y sistemas, se articula sobre todo diciendo que con ello se aplaza o se olvida para siempre la resolución de los problemas fundamentales. En definitiva, según él, la energía que debería primar en el estudio filosófico se pone ahora en algo que no son las cuestiones esenciales del debate filosófico (MacIntyre 1994, 333-349).

Sin embargo, hay además algo también importante para MacIntyre: la práctica de la filosofía analítica, al ceñirse al examen de inferencias procedimentales, es una herramienta inútil para refutar el emotivismo que asola la sociedad y el liberalismo que la intenta sustentar. MacIntyre insiste mucho en este punto, porque esta actitud emotivista limita poderosamente la resolución de las discrepancias morales que nuestro autor ha descrito con tanto acierto.

Sólo en raras ocasiones la filosofía analítica puede producir [...] resultados concluyentes de tipo negativo. Puede mostrar en algunos casos que hay demasiada incoherencia e inconsistencia en una posición para que una persona razonable siga defendiéndola. Pero no puede jamás establecer la aceptabilidad racional de una posición particular en los casos en que cada una de las alternativas rivales [...] es lo suficientemente coherente (MacIntyre 1991, 115; Ruiz Ariola 2000, 130-131).

Según MacIntyre, la filosofía analítica se puede articular en cuatro características que la definen en el contexto de su enseñanza e investigación (MacIntyre 1992, 202-204; Ruiz Arriola 2000, 131-132). La primera se puede enunciar diciendo que este tipo de filosofía contiene una gran destreza lógica y conceptual, pero al precio de limitar mucho el número de participantes en el debate filosófico por la correspondiente sanción burocrática de las personas cualificadas para ello. La segunda característica es, para MacIntyre, el tratamiento pormenorizado de cuestiones muy concretas o puntuales, lo que hace que el debate se aleje del tema central de la comunidad, que es el conjunto de aquellos asuntos que conciernen a la vida común. La tercera es la nota más inaceptable, según él, de la filosofía analítica: que se funda- 
menta en un acuerdo meramente formal y no en un compromiso vital con las tesis defendidas. La última es un resumen de las anteriores y representa un corolario de lo expresado en este párrafo: en este tipo de hacer filosofía se observa un compromiso ideológico previo que, significativamente, es externo al debate mismo para llegar un acuerdo.

Como se ve, la crítica de MacIntyre a la forma en que extensamente se enseña filosofía en una gran mayoría de universidades anglosajonas es nuclear. Se está enseñando, se estudia y se investiga en profundidad sobre aspectos concretos; pero no se enseña a vivir la verdad. Y, sin embargo, esto último es el corazón de la enseñanza filosófica.

El gran problema, para MacIntyre, no está tanto en que la filosofía analítica contradiga sus propias tesis sobre cómo debe ser la filosofía. El aspecto más preocupante es el entronque de ésta con los saberes humanistas y, consecuentemente, con el actuar de la sociedad (Ruiz Arriola 2000, 133). Es en este contexto donde la especialización que ha padecido en los últimos tiempos la tarea de filosofar sufre las consecuencias más letales. La erudición filosófica se ha transformado en un fín en sí mismo, y se ha relegado al olvido la búsqueda de la vida buena, cómo vivir bien. Lo dramático de todo este proceso es que, con estos esquemas conceptuales especializados que excluyen al público lego del debate filosófico -la filosofía ha dejado de ser vida para las gentes-, el empobrecimiento ha sido mutuo: la sociedad ha perdido una guía clara y la filosofía ha dejado de ser relevante (e influyente) para la vida social y su desarrollo (MacIntyre 1987b, 25; 1992, 213).

Transcribimos a continuación unas palabras del filósofo británico que pretenden clarificar muchas de las ideas que se han ido trasmitiendo en este apartado.

Un filósofo puede estar en dos tipos muy diferentes de relación con la sociedad más amplia de la que es parte. En ciertos tipos de situación social puede ser un activo participante en los foros de debate público, y criticar en ocasiones los criterios de racionalidad establecidos y socialmente compartidos, pero apelando incluso en esas ocasiones a los criterios compartidos por un público generalmente educado, o que, al menos, le son accesibles a dicho público. Y éste puede ser el caso aun cuando el filósofo asuma el papel de crítico radical, como hizo 
Platón. Pero cuando el profesionalizado filósofo académico hace de la discusión racional de cuestiones de importancia fundamental la prerrogativa de una élite académica con habilidades técnicas certificadas, que usa un vocabulario y escribe en géneros que son inaccesibles a los que están fuera de esa élite, los excluidos son propensos a responder rechazando la racionalidad de los filósofos. En los foros de la vida popular la eficacia retórica en la persuasión y en la manipulación prevalece frente a la argumentación racional (MacIntyre 1992, 213).

Llama la atención lo certero del comentario de MacIntyre, cuando uno ve lo que está sucediendo en la vida académica y política de nuestra sociedad en este comienzo del siglo XXI.

\section{El olvido de la educación para la sabiduría y el carácter}

Para MacIntyre, el abandono de los fines de la educación como los que se señalan en el título de este apartado, representa todo lo que conlleva la formación liberal. Nuestro autor es claro al señalar que la neutralidad filosófica pertenece al reino de la fantasía. La comunidad humanista está llena de desacuerdos y enfoques emotivistas que dificultan el discurso racional. Además, el facticismo ha invadido completamente la ciencia experimental; la eficiencia se ha dejado a las ciencias sociales y a las disciplinas técnicas agrupadas en la más exitosa de todas ellas, la economía.

MacIntyre siempre ha dado gran importancia a esto último que señalamos, quizá fruto de sus estudios de la doctrina marxista y de las ciencias sociales. El filósofo británico ve que la mercantilización de la sociedad, ocurrida en el proceso ilustrado, ha originado la adopción de la organización del mercado como principio rector de la política, que clásicamente se consideraba un saber moral en cuanto que se encargaba de la administración de las cosas y el gobierno de los hombres (Ruiz Arriola 2000, 134-135).

La crítica de MacIntyre a la modernidad viene a decir con nitidez que la relegación de la moralidad al ámbito privado ha acarreado, alternativamente, una impronta economicista en la consideración de todas las disciplinas sociales. Todo ello ha tenido asimismo un efecto perverso en el individuo. Porque, en vez de ayudarle a pensar por sí mismo -que era el principal 
cometido de la universidad liberal-, todos los cambios de criterio que acabamos de señalar han terminado por inhibir esta capacidad, bien por el camino del relativismo moral, bien por la propia especialización fáctica o bien por una educación que favorece una racionalidad incapaz de dar respuesta a lo esencial de la vida humana.

La formación liberal intenta promover y desarrollar en los alumnos unas disposiciones que les permitan alcanzar los bienes externos al mínimo costo y con el máximo beneficio (Ruiz Arriola 2000,138). A estas habilidades les ha llamado MacIntyre cualidades de eficiencia:

Son aquellas que, en las circunstancias en las que una persona concreta se encuentra, le permiten tanto identificar los medios que serán eficaces para conseguir dichos bienes [externos], como ser eficaz al utilizar esos medios para conseguir los [citados] bienes [externos] (MacIntyre 1984, 49).

Entre estas cualidades de eficiencia, MacIntyre se ha detenido de forma muy especial en los rasgos del carácter, que nuestro autor entiende como expresiones de las pasiones naturales o de las disposiciones para dominar o limitar su efecto destructivo en nuestra conducta. Pues bien, nuestro pensador nos dirá que el establecimiento inapelable de la razón instrumental, que está en el centro de la formación de la universidad liberal, nos va a permitir satisfacer las pasiones naturales. Y esto convive con una concepción ética minimalista y deontológica, que tiene como límite específico la propia ambición personal y la satisfacción de los propios deseos.

La unión de estas dos corrientes liberales dominantes -que pueden enunciarse como el desarrollo de los propios talentos, por una parte, y la restricción de su ejercicio por unas normas morales carentes de justificación racional, por otra- da lugar a lo que MacIntyre ha indicado en su determinación de la universidad liberal, a saber, que en ella se da la presencia de corrientes contrapuestas subyacen bajo unas explicaciones técnicas que tienen apariencia de racionalidad. Nuestro autor nos hará ver que, como colofón permitido y bendecido por unos criterios de claro corte utilitarista, todo se disfraza de enseñanza de unos cursos de ética aplicada, que para él no son nada más que una propaganda inservible (Bloom 1987, 61; MacIntyre 1992, 278-279). 
Según MacIntyre, el verdadero motor de la universidad liberal en el área socio-política es la denominada pleonexia,

que no es ni más ni menos que una simple avaricia, el actuar sólo para tener más (MacIntyre 1994, 121).

Siguiendo a Aristóteles, el filósofo escocés continúa describiendo la crematística como una disposición del afán de vivir, pero no del afán de vivir bien (Aristóteles 1989, 1257b16),

[de tal modo que] el rasgo del carácter mostrado y desarrollado por tal actividad no se dirigirá de acuerdo con ese medio que guía hacia el telos de la vida buena para los seres humanos (MacIntyre 1994, 121-122).

En definitiva, estamos ante otra de las paradojas de esa formación de la universidad liberal, que tan claramente nos intenta hacer ver MacIntyre: por una parte, sirve a los fines del poder, la posición social y la riqueza, y por otro, pretende poner todo eso al servicio de un bien común que se ha camuflado y es, por tanto, inexistente.

\section{La justificación de la universidad liberal}

Ruiz Arriola ha expuesto con acierto lo que pretendemos decir en este último apartado: «La Universidad Liberal no escapa a esta dinámica circular que caracteriza a las tradiciones. Si la Universidad goza hoy de prestigio en algunos sectores de la sociedad, no es por su capacidad de enriquecer -en el sentido amplio del término- a sus alumnos ni a la sociedad misma. Como se ha intentado dejar claro a lo largo de este diagnóstico, la Universidad Liberal, supuestamente neutral por sus principios ilustrados así como por su misión institucional de búsqueda desinteresada de la verdad, entra en el juego del orden liberal. Como todas las instituciones y actividades dentro de ese orden, se justifica en términos de la tecno-estructura dominante: la preparación de expertos para la gestión del orden social y económico» (Ruiz Arriola 2000, 139). 
En una línea de crítica, con esa tecno-estructura dominante de la sociedad que genera la universidad liberal y con interesantes analogías a todo lo que venimos comentando, puede resultar también interesante consultar la entrevista que hicieron al profesor Juan Bautista Fuentes en el año 2005 (Romero Cuadra y Hermoso Félix 2005, 181-199).

En suma, parece claro que la universidad liberal se convierte, en gran medida, en un aparato burocrático que sanciona un saber técnico, y que se justifica y goza de prestigio porque, como nos dice Long, tiene «"poder” para conferir títulos académicos que faciliten la entrada en los empleos avanzados» (Long 1992, 179; Ruiz Arriola 2000, 141).

MacIntyre no critica en este punto el entrenamiento laboral en sí mismo, sino, más bien, que dados los fundamentos en los que se apoya la universidad liberal, está impedida para cumplir integralmente su misión educativa. Su dinámica le lleva a formar alumnos en profesiones y caminos vocacionales que van compitiendo entre sí y que carecen de cualquier orden y jerarquía en aspectos fundamentales para la acción humana y la mejor vida para el hombre.

[...] aquello en que muchos de nosotros hemos sido educados no es un modo coherente de pensar y juzgar, sino algo construido a partir de una amalgama de fragmentos sociales y culturales heredados, tanto de las diferentes tradiciones de las que nuestra cultura originariamente se derivaba [puritana, católica, judía)] como de los diferentes momentos y aspectos del desarrollo de la modernidad [la ilustración francesa, la ilustración escocesa, el liberalismo económico decimonónico, el liberalismo político del siglo veinte] (MacIntyre 1994, 19-20).

Viendo las cosas en profundidad, la justificación de la universidad liberal se resume en que no se va al fondo porque el entorno cultural ha querido asumir plenamente los presupuesto liberales de la modernidad. Dejemos que sean otra vez las propias palabras de MacIntyre las que nos ofrezcan esta reflexión en una cita larga, pero muy ilustrativa.

Uno de los rasgos más llamativos de los órdenes políticos modernos es su carencia de foros institucionalizados dentro de los cuales estos desacuerdos 
fundamentales puedan investigarse y esbozarse sistemáticamente, así como la ausencia de intento alguno para resolverlos. Con frecuencia, los mismos hechos del desacuerdo pasan inadvertidos, disfrazados por una retórica de consenso. Y si en algún asunto complejo y singular -como en las disputas sobre la guerra del Vietnam o los debates sobre el aborto- las ilusiones de un consenso sobre las cuestiones de justicia y de racionalidad práctica se encuentran de momento rotas, las expresiones de desacuerdo radical se institucionalizan de tal forma que se sustrae dicho asunto singular del telón de fondo de las diferentes e incompatibles creencias a partir de las cuales semejantes desacuerdos irrumpen. Este hecho sirve para prevenir, en la medida de lo posible, que el debate se extienda a los principios fundamentales que informan a aquellas creencias de fondo (MacIntyre 1992, 20).

En el texto que acabamos de citar, hemos señalado en cursiva una frase que nos parece clave en el análisis que hace MacIntyre del entorno formativo de la sociedad que vivimos. Entre esos foros que cita aquí MacIntyre, ¿no esperaría uno encontrar la institución universitaria, como lugar privilegiado para esos debates que buscan la racionalidad?

En definitiva, para nuestro autor, la propia universidad liberal está sumergida en un orden técnico-instrumental que se ha desarrollado en la sociedad contemporánea. Dicho orden es el semillero de una élite liberal que, con su creciente burocracia y su pericia, pretende manipular la sociedad a la que la propia universidad pretende servir. Y esto, según MacIntyre, lo ha logrado exitosamente.

\section{Referencias}

Aristóteles. 1989. Política. Madrid: Centro de Estudios Políticos y Constitucionales. Becker, Gerhold K. 1994. "Unity and the University: The Neo-Humanist Perspective in the Age of Post-Modernism.” International Philosophical Quarterly 34:177-189. Bell, Daniel. 1992. Las contradicciones culturales del capitalismo. Madrid: Alianza. Bello Rodríguez, Hernando José, y José Manuel Giménez Amaya. 2018. Valoración ética de la modernidad según Alasdair MacIntyre. Pamplona: EUNSA.

Bloom, Allan. 1987. The Closing of the American Mind. New York: Simon \& Schuster. Bowen, James. 1986. Historia de la educación occidental: t. II: la civilización europea, siglos VI a XVI. Barcelona: Herder. 
Giménez Amaya, José Manuel. 2012. «La fragmentación y “compartimentalización” del saber según Alasdair MacIntyre». En Sapienza é Libertá: studi in onore del Prof. Lluís Clavell, a cura di Miguel Pérez de Laborda, 193-202. Roma: Edizioni Santa Croce.

Giménez Amaya, José Manuel, y Sergio Sánchez-Migallón. 2011. Diagnóstico de la Universidad en Alasdair MacIntyre. Génesis y desarrollo de un proyecto antropológico. Pamplona: EUNSA.

Kerr, Clark. 1963. The Uses of University. Cambridge, Massachusetts: Harvard University Press.

Long, Edward LeRoy. 1992. Higher Education as a Moral Enterprise. Washington, D. C.: Georgetown University Press.

Lutz, Christopher S. 2009. Tradition in the Ethics of Alasdair MacIntyre: Relativism, Thomism, and Philosophy. Lanham, Maryland: Lexington Books.

Llano, Alejandro. 1988. La nueva sensibilidad. Madrid: Espasa Universidad.

MacIntyre, Alasdair. 1983. “The Indispensability of Political Theory.” In The Nature of political Theory, edited by David Miller, and Larry Siedentop, 17-33. Oxford: Clarendon Press.

MacIntyre, Alasdair. 1984. After Virtue: A Study in Moral Theory (2nd Edition). Notre Dame, Indiana: University of Notre Dame Press.

MacIntyre, Alasdair. 1987a. Tras la virtud. Barcelona: Crítica.

MacIntyre, Alasdair. 1987b. “The Idea of an Educated Public.” In Education and Values (The Richard Peters Lectures, delivered at the institute of Education, University of London, Spring Term, 1985), edited by Graham Haydon, 15-36. London: London University Press.

MacIntyre, Alasdair. 1989. "The relationship of Philosophy to History: PostScript to the Second Edition of After Virtue.” In After Philosophy: End or Transformation?, edited by Kenneth Baynes, James Bohman, and Thomas McCarthy, 381-422. Cambridge, Massachusetts: MIT Press.

MacIntyre, Alasdair. 1991. "Incommensurability, Truth and the Conversation Between Confucians and Aristotelians About the Virtues." In Culture and Society East-West Philosophic Perspectives, edited by Eliot Deutsch, 104-122. Honolulu: University of Hawaii Press.

MacIntyre, Alasdair. 1992. Tres versiones rivales de la ética. Madrid: Rialp.

MacIntyre, Alasdair. 1994. Justicia y racionalidad. Pamplona: EIUNSA.

MacIntyre, Alasdair. 2012. Dios, filosofía, universidades: historia selectiva de la tradición filosófica católica. Granada: Editorial Nuevo Inicio.

MacIntyre, Alasdair. 2017. Ética en los conflictos de la modernidad: sobre el deseo, el razonamiento práctico y la narrativa. Madrid: Rialp. 
Martínez-Echevarría Castillo, Íñigo. 2010. La relación de la Iglesia con la Universidad en los discursos de Juan Pablo II y Benedicto XVI: una nueva aproximación jurídica. Roma: Edizioni Santa Croce.

Pieper, Joseph. 1983. El ocio y la vida intelectual. Madrid: Rialp.

Rodríguez Duplá, Leonardo. 2000. «Las aporías del liberalismo». En Las ideologías al final de siglo: perspectivas desde el pensamiento cristiano, 31-43. Salamanca: Ediciones Universidad de Salamanca.

Romero Cuadra, José Luis, y María Jesús Hermoso Félix. 2005. «Entrevista al prof. Juan Bautista Fuentes: filosofía, política y metapolítica». Nexo. Revista de Filosofía 3:181-199.

Ruiz Arriola, Claudia. 2000. Tradición, universidad y virtud: filosofía de la educación superior en Alasdair MacIntyre. Pamplona: EUNSA.

Saranyana, Josep-Ignasi. 2007. La filosofía medieval: desde sus orígenes patrísticos hasta la escolástica barroca, Pamplona: EUNSA.

Winchester, Ian. 1986. “The Future of a Mediaeval Institution: The University in the Twenty-first Century." In Universities in Crisis: A Mediaeval Institution in the Twenty-first Century, edited by William A. W. Neilson, and Chad Gaffield, 269-290. Montreal, Québec: The Institute for Research on Public Policy. 